\title{
Continuum spectroscopy of light nuclei
}

\author{
R. J. Charity for the HiRA Collaboration
}

Department of Chemistry, Washington University,

St. Louis MO 63130, USA

\begin{abstract}
Resonance spectroscopy of light nuclei is discussed with emphasis on the invariant-mass measurements performed with the HiRA detector. For three-body exit channels, we consider the exact conditions necessary such that the decay can be described as either sequential or prompt. However experimentally, we find some cases where the decay is intermediate between these two limits. Finally, two-proton decay from isobaric analog states is discussed.
\end{abstract}

Light nuclei near the drip lines have very few particle-bound levels, with most of their states being in the continuum. Of course those beyond the drip lines have all their levels in the continuum including their ground states. Thus the study of continuum spectroscopy is quite important for these light nuclei. There are many techniques to study these continuum states, however, in this work we will consider just the invariant-mass method and discuss results obtained with the HiRA array [1] at the the National Superconducting Cyclotron Laboratory at Michigan State University and the Texas A\&M University cyclotron.

It is often assumed that continuum states which are associated with binary exit channels are dominant. However, three-body and high-order exit channels are quite common in light nuclei. For example in the ${ }^{9} \mathrm{C}+{ }^{9} \mathrm{Be}$ reaction at $E / A=70 \mathrm{MeV}$ studied with the HiRA detectors [17], we have identified 42 resonances in the continuum which decay into exit channels containing charged particle. These resonances are continuum states in ${ }^{5,6,7} \mathrm{Li}$, ${ }^{6,7,8} \mathrm{Be},{ }^{7,8,9} \mathrm{~B}$, and ${ }^{8,9,10} \mathrm{C}$ nuclei. Of these, only half were associated with 


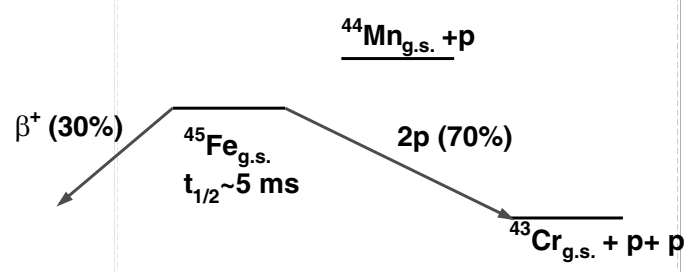

Figure 1: Level scheme for the decay of ${ }^{45} \mathrm{Fe}$.

binary exit channels with the remainder from three, four, and five-body decays. Thus these higher-order decays are quite common and will be the subject of the rest of this paper.

These higher-order decay channels can be associated with either a prompt decay scenario where all of the exit-channel particles are emitted at the same time or the disintegration could be sequential where the decay proceeds through a binary decay channel producing one or more intermediate states which are themselves resonances and subsequently decay.

As an example of three-body decay, Goldansky considered prompt twoproton emission from ground states in 1960 [3]. He envisaged this process occurring for systems where no intermediate state in the one-proton daughter is energetically available, so the system is forced to decay by a prompt two-proton emission. As an example, the level scheme for the decay of ${ }^{45} \mathrm{Fe}_{\text {g.s. }}$ is shown in fig 1 . Here the ${ }^{44} \mathrm{Mn}_{\text {g.s. }}$. intermediate is not available for one-proton decay so the only allowed particle-decay mode is a two-proton emission. This state also has a smaller $\beta$-decay branch.

For odd- $Z$ parents, the pairing interactions pushes the mass of the even- $Z$ one-proton daughter down always making it energetically accessible in these light systems. Thus "Goldansky" type ground-state two-proton emitters must have even- $Z$. Also the Goldansky's scheme assumes that if a oneproton decay channel is available, the level will chose that branch rather than a prompt two-proton channel for decay.

Light nuclei beyond the proton drip line are more complicated than the Goldansky scheme as the small Coulomb barriers can give rise to substantial widths for the intermediate states. For a true sequential three-body decay, the intermediate state should be narrow (long lived) so that there is a time 


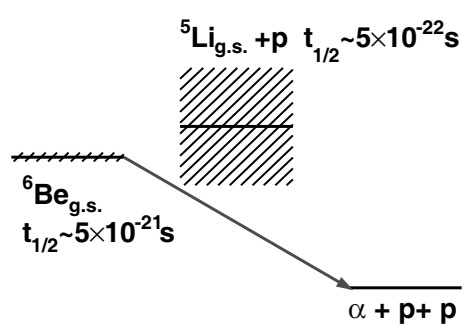

Figure 2: Level scheme for the decay of ${ }^{6} \mathrm{Be}$.

separation in the two binary emission steps allowing no interactions between the particles produced in these two steps. In the limit of a very large width for this intermediate state, the decay will turn into a prompt decay. For potential sequential two-proton emitters where the energy of the first proton is of the same magnitude as the width of the intermediate state, the decay is called democratic [4] and considered prompt as the first proton will not have traveled any distance before the second proton is emitted. One should be careful of the term democratic decay as it has also been used to describe decays where the momentum correlations between the final fragments follow phase-space considerations which is quite different from the present usage.

The level scheme for the decay of ${ }^{6} \mathrm{Be}$, the lightest two-proton groundstate emitter, is shown in fig. 2. One can see that most of the strength of the ${ }^{5} \mathrm{Li}_{\text {g.s. }}$. intermediate state is energetically inaccessible so it has been considered as a Goldansky decay by some people. However as this intermediate state is very wide, decays though its low-energy tail are still permissible. But even in this case, the width of this state is of the same order as the energies of the allowed single-proton decays, so this state must be considered as democratic and prompt. The pairing effect is also important for groundstate democratic decay. The valence protons for odd- $Z$ systems sit higher up in the Coulomb well than their more-bound even- $Z$ neighbors and thus have much larger widths, Therefore even- $Z$ parents are required in order to have wide odd- $Z$ intermediate states.

Figure 3 shows a portion of the chart of nuclides for light systems highlighting the known ground-state two-proton and two-neutron emitters. It is not surprising from the above arguments that all two-proton emitters are even- $Z$. From a similar logic, one expects the ground-state two-neutron 


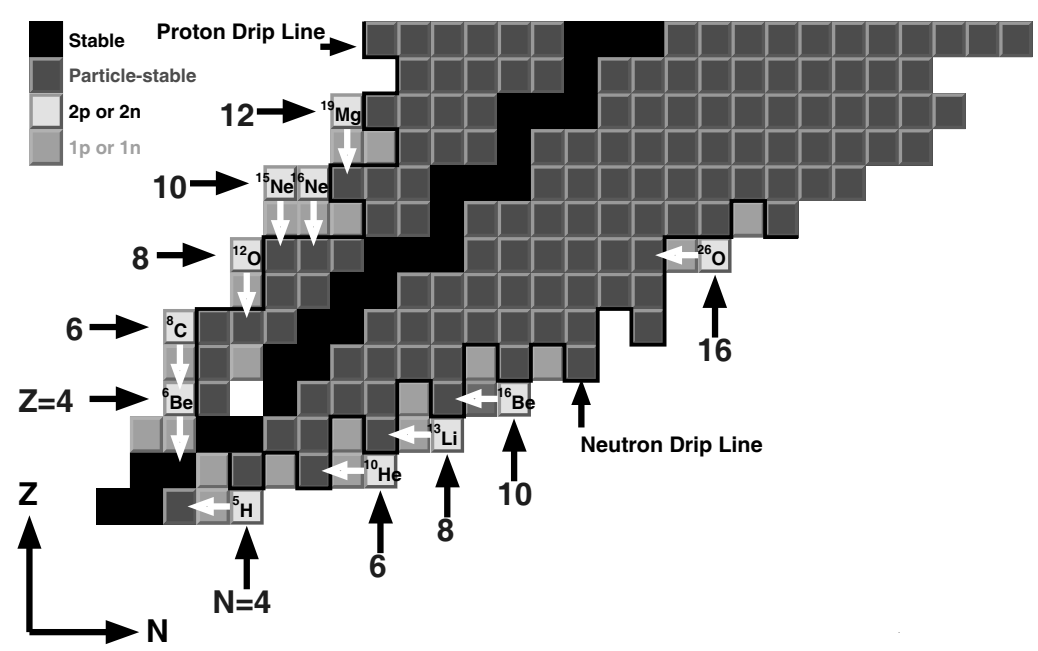

Figure 3: A portion of the chart of nuclides showing the location of known groundstate two-proton and two-neutron emitters in light systems. Each two-nucleon emitter is labeled and a white arrow indicates it decay.

emitters on the other side of the chart of nuclides to be even $N$. Important information about these higher-order decays is contained in the momentum correlations between the final products. In three-body decay, with three momentum components for each fragment, there are nine degrees of freedom in total. However, three degrees of freedom are constrained by momentum conservation and a further one from energy conservation. In addition any overall rotation of the three momentum vectors is not of interest, reducing the problem by a further three degrees of freedom associated with the three Euler angles of rotation. This leaves us finally with two degrees of freedom. Thus all three-body decays have a two-dimensional distribution describing their momentum correlations. One is of course free to choose which two parameters to use.

One choice of parameters is obtained from the Jacobi Y hyperspherical coordinates. Here we define an energy parameter $E_{x} / E_{T}$ and angle parameter $\theta_{k}$ where $E_{x}$ is the relative energy between the core and a proton, $E_{T}$ is the total decay energy, and $\theta_{k}$ is the angle between the proton-core relative velocity and the center-of-mass velocity of the other proton. Figure 4 compares measured two-dimensional correlations for (a) ${ }^{6} \mathrm{Be}_{\text {g.s. }}$. [5] a prompt two-proton emitter and (b) the $5.3-\mathrm{MeV}$ excited state of ${ }^{10} \mathrm{C}[6]$, a sequential two-proton emitter. 

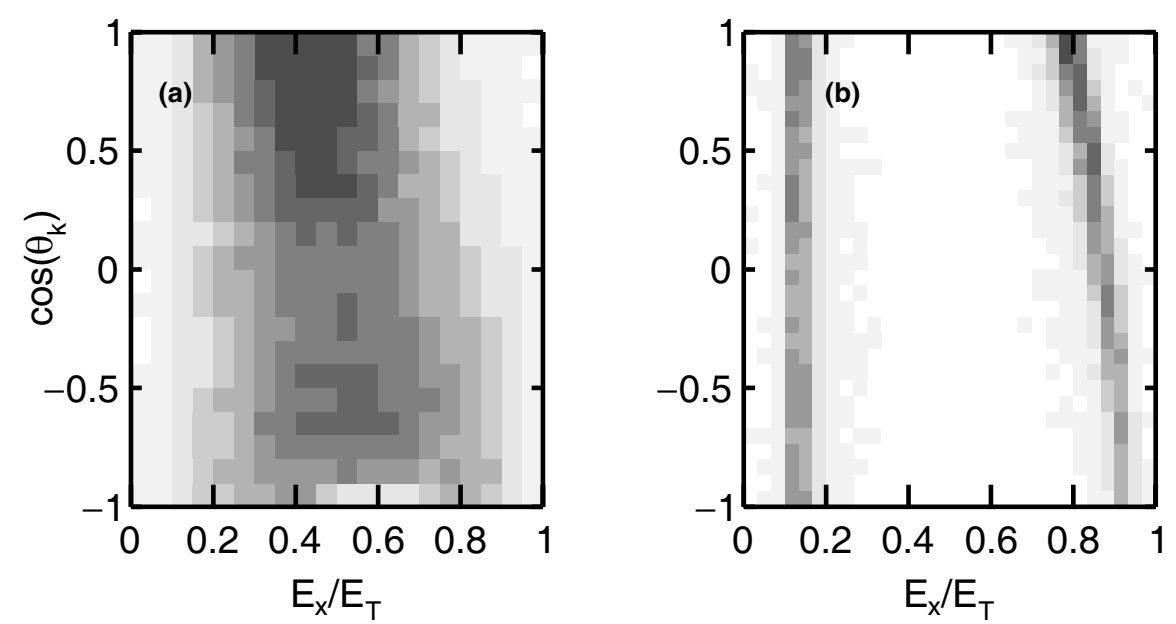

Figure 4: Jacobi $\mathrm{Y}$ correlation plots measured for the two-proton decay of (a) ${ }^{6} \mathrm{Be}_{\text {g.s. }}$ and (b) the $5.3-\mathrm{MeV}$ excited state of ${ }^{10} \mathrm{C}$.

One can see clear differences between the correlations for prompt and sequential decay. The sequential decay of fig. 4(b), which is easiest to understand, consists of two narrow ridges that correspond to the two emitted protons. The low-energy ridge, which is independent of the angle $\theta_{k}$ is the second emitted proton. For the high-energy ridge (first proton), $E_{x}$ (the relative $p$-core energy) depends on $\theta_{k}$ due to the recoil imparted by the second proton. In contrast, the prompt decay of fig. 4(a) has strength in a single, more-spread-out region of correlation space.

Systematics of the projection of the correlations on the energy axis are shown in fig. 5 for three measured prompt-two-proton emitters: ${ }^{6} \mathrm{Be}_{\text {g.s. }}$ [5], ${ }^{16} \mathrm{Ne}_{\text {g.s. }}$ [7], and ${ }^{45} \mathrm{Fe}_{\text {g.s. }}$ [8]. The results for ${ }^{45} \mathrm{Fe}$ are not from the HiRA group, but were obtained with an optical time projection chamber [8]. All distributions have similar shape with a peak at $E_{x} / E_{T} \sim 0.5$, i.e., equal energy protons. This result was originally predicted by Goldansky [3] who indicated that the two-proton emission probability must be proportional to the product of two barrier penetration factors $P(E)$, i.e.

$$
P_{2 p} \propto P\left(E_{x}\right) P\left(E_{T}-E_{x}\right)
$$

if the two protons are emitted simultaneously. This product peaks for equal energy protons. As one becomes more sub-barrier, the penetration factors increase more rapidly with energy and thus the $E_{x} / E_{T}$ distribution should become narrower. This is clearly demonstrated in fig. 5 where the $E_{x} / E_{T}$ width decreases quite substantially with increasing $Z$ (larger Coulomb bar- 


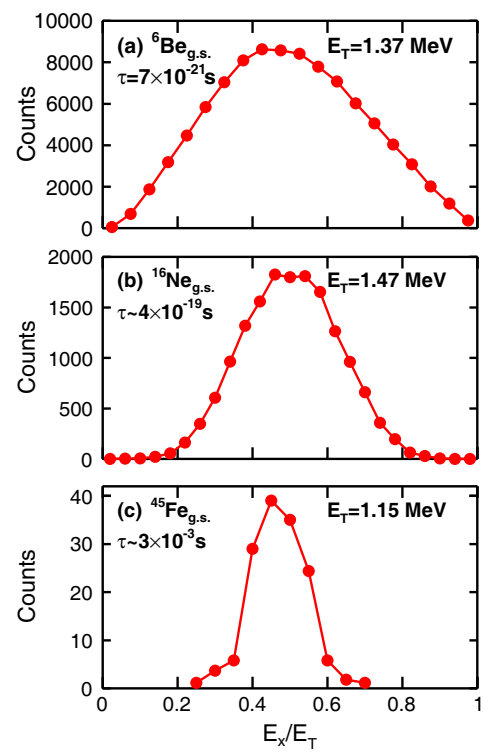

Figure 5: Measured distributions of $E_{x} / E_{T}$ for three ground-state two-proton emitters.

riers for the proton emission). Note the total decay energy of the three systems in fig. 5 are all similar and thus it is only the change in the height of the Coulomb barriers that are affecting the degree of sub-barrier emission in these three cases. ${ }^{45} \mathrm{Fe}$ with the largest Coulomb barrier is most subbarrier and has the narrowest $E_{x} / E_{T}$ distribution and the longest lifetime.

Theoretical predictions of these momentum correlations must include the initial-state correlation and the final-state interactions as the fragments move apart. The latter has contributions from the spin-singlet $p-p$ interactions and the long-range three-body Coulomb interaction. In the three-body cluster model developed by Grigorenko et al [9], the wavefunction of the three-body system is solved in hyperspherical coordinates and matched to approximate outgoing waves associated with the Coulomb interaction. Further refinements have been made in more recent years [7]. This model reproduces the measured correlations in the ground and first-excited $\left(J=2^{+}\right)$ states of ${ }^{6} \mathrm{Be}[5]$ very well. More recently it has been applied to the twoproton decay of the ground state of ${ }^{16} \mathrm{Ne}$ where the long-range Coulomb final-state interactions are much stronger [7]. The calculations were able to reproduce the experimental data quite well as long as the three-body Coulomb interactions are considered out to distances of $\sim 100000 \mathrm{fm}$. This results validates these calculations for use with heavier two-proton emitters. 


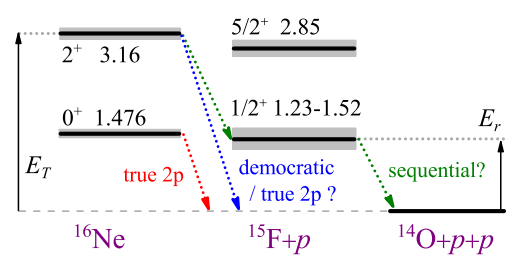

Figure 6: Level scheme of ${ }^{16} \mathrm{Ne}$ and the states associated with its decay.
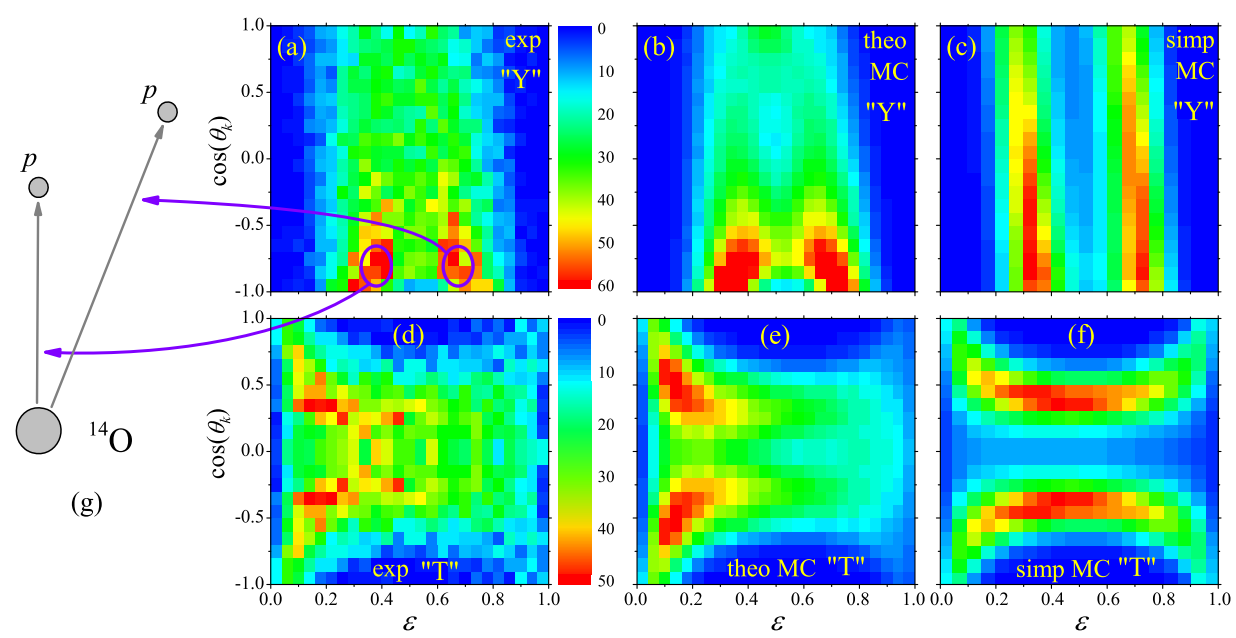

Figure 7: Momentum correlations for the decay of the first excited state of ${ }^{16} \mathrm{Ne}$. The correlations $(a-c)$ are in the Jacobi Y representation while (d-f) are in the Jacobi $\mathrm{T}$ representation. The experimental results (a,d) can be compared to the prediction of the three-body cluster model (b,e), and a sequential calculation (c,d). The prominent configuration indicated by the circles in (a) is shown in (g). Here $\varepsilon=E_{x} / E_{T}$.

Although the ground states of ${ }^{6} \mathrm{Be}$ and ${ }^{16} \mathrm{Ne}$ are prompt two-proton emitters, it is interesting to determine the nature of their excited states. The level scheme of ${ }^{16} \mathrm{Ne}$ and its potential ${ }^{15} \mathrm{~F}$ intermediate states are shown in fig. 6. For the $J=2^{+}$first excited state, neither the Goldansky or the democratic conditions apply. The ground state of ${ }^{15} \mathrm{~F}$ is energetically accessible by one-proton decay and the kinetic energy of such a proton is large compared to the width of this state. So does this states decay by sequential two-proton decay? The correlations measured for this state are shown in fig. 7(a,d) [10]. In addition to the Jacobi Y representation, we also show the Jacobi $T$ representation where here $\varepsilon=E_{x} / E_{T}$ and $E_{x}$ is now the relative energy between the two protons. In the Jacobi $\mathrm{T}$ representation, 


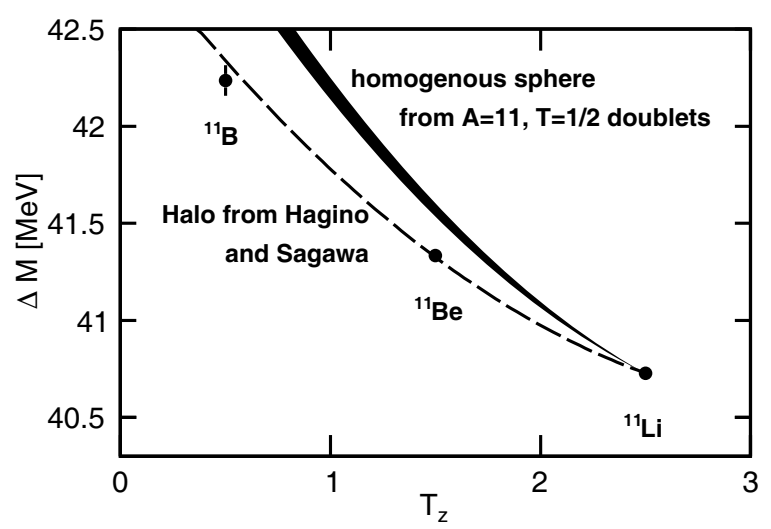

Figure 8: Mass excesses of the three known members of the $A=11$ sextet plotted as a function of isospin projection. The solid band shows the prediction where the Coulomb energy is determined with a homogeneous-sphere assumption with the radius extracted from the $T=1 / 2 A=11$ doublet. The dashed curve shows the prediction using the halo wavefunction of Hagino and Sagawa [16].

the angle $\theta_{k}$ is now the angle between the relative velocity vector between the proton pair and the center-of-mass velocity of the core.

In comparison, fig. $7(\mathrm{c}, \mathrm{f})$ shows the predicted pattern expected for a sequential decay which displays a two-ridge pattern similar to that obtained for ${ }^{10} \mathrm{C}^{*}$ in fig. 4(b). However, the experimental distribution only shows the two-ridge structure for $\cos \left(\theta_{k}\right) \sim-1$ in the Jacobi Y representation of fig. 7(a), i.e., where the relative angle between the two protons is small and thus large $p-p$ final-state interactions are expected. This behavior is reproduced by the three-body cluster-model prediction in fig. $7(\mathrm{~b}, \mathrm{e})$. In this model one finds the existence of both prompt and sequential decay paths that interfere and produce the strange correlation pattern. A similar behavior is found in the ${ }^{6} \mathrm{Be}$ continuum at excitations of 6 to $9 \mathrm{MeV}$ [5]. Clearly the exact conditions for pure sequential decay have yet to be determined.

So far we have only considered two-proton emission from ground and low-lying excited states. Two-proton emission has also been observed from isobaric analogs of ground-state two-proton emitters. These include the isobaric analog of ${ }^{8} \mathrm{C}_{\text {g.s. }}$ in ${ }^{8} \mathrm{~B}[11,12]$ and the isobaric analog of ${ }^{12} \mathrm{O}_{\text {g.s. }}$ in ${ }^{12} \mathrm{~N}$ [13]. Both of these two cases can be considered as extensions of the Goldansky argument, i.e, single-proton emission violates either energy or isospin conservation leaving two-proton decay as the only channel satifiying both conservation laws. 
Finally, we have also observed two-proton emission in an analog of the two-neutron-halo state ${ }^{11} \mathrm{Li}_{\text {g.s. }}$. A peak associated with the double isobaric analog of ${ }^{11} \mathrm{Li}_{\text {g.s. }}$ in ${ }^{11} \mathrm{~B}(T=5 / 2)$ was found in the $2 p+{ }^{9} \mathrm{Li}$ exit channel created with the ${ }^{12} \mathrm{Be}+{ }^{9} \mathrm{Be}$ reaction [14]. To the extent that isospin is conserved, this state should have the same structure as ${ }^{11} \mathrm{Li}_{\text {g.s. }}$ except that two of the neutrons have been turned into protons and therefore there are components of the wavefunction where the halo contains one or two protons. Figure 8 shows the evolution of the mass excess for the $T=5 / 2$ sextet associated with these states. In addition to the new $T_{z}=1 / 2{ }^{11} \mathrm{~B}$ member, the $T_{z}=3 / 2{ }^{11} \mathrm{Be}$ member is also known [15]. The increase in the mass excess with decreasing $T_{z}$ is due to the larger Coulomb energies of these more proton-rich analogs. The predicted evolution when the Coulomb energy is obtained from a homogeneous-sphere assumption produces a much larger $T_{z}$ dependence than observed. If however one allows for a halo structure and uses the halo wavefunction predicted by Hagino and Sagawa [16], then the mass excesses can be reproduced (dashed curve). Of course putting protons in an extended halo structure give rise to smaller Coulomb energies and masses. Thus the extent of the halo structure of ${ }^{11} \mathrm{Li}$ is also contained in the masses of its analogs.

In summary, the field of continuum spectroscopy is quite exciting for these light nuclei. Momentum correlations have proved to be a powerful tool in determining the sequential and prompt aspects of three-body decay. However the exact nuclear structure necessary for sequential and prompt decays have yet to be determined. While this contribution has focused on two-proton emission, there are many other 3-body and 4-four decays observed in light nuclei [17]. In addition the binary decays are also of interest, for example in astrophysics [2].

This work is supported by the U.S. Department of Energy, Office of Science, Office of Nuclear Physics under Award No. DE-FG02-87ER-40316. The HiRA group acknowledges the considerable theoretical support of Leonid Grigorenko.

\section{References}

[1] M. Wallace et al., Nuclear Instruments and Methods A 583 (2007) 302.

[2] L.G. Sobotka et al., Phys. Rev.C 87 (2013) 054329.

[3] V.I. Goldansky, Nucl. Phys. 19 (1960) 482. 
[4] O.V. Bochkarev, L.V. Chulhov, A.A. Koreninniicov, E.A. Kuz'min, I.G. Mukha, and G.B. Yankov, Nucl. Phys. A 505 (1989) 215.

[5] I.A. Egorova, et al., Phys. Rev. Lett. 109 (2012) 202502.

[6] R.J. Charity et al., Phys. Rev. C 80 (2009) 024306.

[7] K.W. Brown et al., Phys. Rev. Lett. 113 (2014) 232501.

[8] K. Miernik et al., Phys. Rev. Lett. 99 (2007) 192501.

[9] L.V. Grigorenko et al., Phts. Rev. Lett 88 (2002) 042502.

[10] K.W. Brown et al., Phys. Rev. C 92 (2015) 034329.

[11] R.J. Charity et al., Phys. Rev. C 82 (2010) 041304.

[12] K.W. Brown et al., Phys. Rev. C 90 (2014) 027304.

[13] M.F. Jager et al., Phys. Rev. C 86 (2012) 011304.

[14] R.J. Charity et al., Phys. Rev. C 86 (2012) 041307.

[15] T. Teranishi et al., Phys. Lett. B 407 (1997) 110.

[16] K. Hagino and H. Sagawa, Phys. Rev. C 72 (2005) 044321.

[17] R.J. Charity et al., Phys. Rev. C 84 (2011) 014320. 